\title{
Home Appliances Control System Based On Android Smartphone
}

\author{
Sachin Kishor Khadke \\ Student, Department of Electronics and Telecommunication NBN Sinhgad School of Engineering, Pune \\ Pune, India \\ Sachinkhadke51@gmail.com
}

\begin{abstract}
The remote appliances control system based on the Android smart phone GUI is designed on Android Smartphone. A user logs into the smart Android phone interface, and clicks the buttons gently to send message commands from the GUI which will be transmitted to home information center through the GSM network. Then the AVR ATmega processor recognizes the specified command, and controls the home appliance switches in the wireless radio frequency manner to achieve remote control of appliances ultimately. This seminar focuses on the design of Android terminal, the communication between PIC and GSM module, the realization of the wireless module device's driver, the difficulty in supplying the appropriate low-voltage DC for MCU and wireless module just by a single live wire. The users can manipulate appliances anytime, anywhere, letting our houses become more and more automated and intelligent. There are some problems in the PC monitor terminal, such as its great bulk, inconvenience to carry, high cost, limited monitoring range and so on. Therefore, it's a good choice to design a terminal based on phone.
\end{abstract}

Keywords: android phone; GSM; Bluetooth; wireless switch; SDK

\subsection{Background}

\section{Introduction}

With the development of technology and the continuous improvement of people's living standard, people are in pursuit of automated, intelligent and convenient home control systems. At present, the PC is used as the remote control terminal for most home control systems; however, there are some problems in the PC monitor terminal, such as its great bulk, inconvenience to carry, high cost, limited monitoring range and so on. Therefore, it's a good choice to design a terminal based on phone. With the popularity of smart phones, particularly, the phone based on Android system is rapidly developed. At its I/O developer conference, Google showed a sneak preview of its Android Home project, which will extend the Android platform into household objects. It means that the remote control based on Android phone will become a mainstream way. After logging into the control interface, users can easily control the lights, TVs and air conditionings anytime, anywhere, which brings great convenience to people and improves the quality of life.

\subsection{Literature Survey}

For this proposed Seminar, following IEEE papers were studied as part of literature survey. Smart Home System for Disabled People Via Wireless Bluetooth gives moneywise concept by using GPRS as the medium to control and monitor home appliances.

Design and Realization of Home Appliances Control System Based on The Android Smartphone present the information about the remote appliances control system based on the Android smart phone is designed and realized. A user logs into the smart phone interface, and clicks the buttons gently to send message commands which will be transmitted to home information Centre through the GSM network. Then the PIC processor recognizes the specified command, and controls the home appliance switches in the wireless radio frequency manner to achieve remote control of appliances ultimately. Exploiting Bluetooth on android mobile devices for home security application present the information about mobile devoice has been integrated into our everyday life. Home automation and security are becoming increasingly prominent features on mobile devoices the mobile devoice and security system communicates via Bluetooth because a short-range-only communication system was desired. With the help of android mobile we can control task such as locking the doors, turning on/off lights remotely. According to kaue, home automation can be useful to those who need to access home appliances while away from their home and can improve the lives of the disabled

\subsection{Motivation}

Disabled people are more likely to be exposed to daily life problems than other healthy people. While deaf people cannot hear the door bell, Alzheimer diseased people can forget the gas open in the kitchen. These are some encountered examples when they 4 are alone at home. With the help of technology, assistant projects can be developed to overcome their difficulties.

Smart homes can also be used to support disabled people, providing safe, secure and empowering environments. The system can allow the user to control many features or automate them. The environment can 
also be monitored by the smart home system to ensure safety and alert people when there is some dangerous situation.

\subsection{Scope}

The users can manipulate appliances anytime, anywhere, letting our houses become more and more automated and intelligent. At present, the PC is used as the remote control terminal for most home control systems [however, there are some problems in the PC monitor terminal, such as its great bulk, inconvenience to carry, high cost, and limited monitoring range and so on. Therefore, it's a good choice to design a terminal based on phone.

\subsection{System overview}

\section{System Architecture}

The system is composed of android mobile terminal, GSM network, home information center and wireless switch. And the architecture of the system is shown in Fig.2.1 at any moment; the GUI in phone allows the user to manually control any of applications in the room. First of all, the action listener should be set for each button which will be clicked to send message command via the GSM network. After reading and parsing the commands, PIC processor controls the wireless module to send the address and data codes to achieve remote control of appliances ultimately.

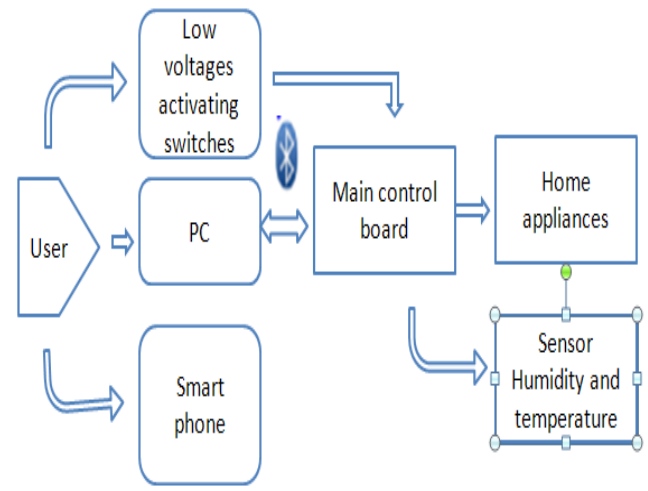

Fig 2.1 Functional Block Diagram of the System

The terminal based on android phone is designed, which contains GUI design, user management and message command sending event. After entering the lighting control interface, users send predefined commands just by touching the appropriate button or the light bulb icon. The user management includes user registration, login, user information storage and its security. The user registration is to create a legitimate user, and give him the appropriate permissions. The current user ID and the message command will be sent to the home information center together. Meanwhile, the user ID must be registered in the home information center, so that the center can identify it effectively. Once the user reveals his information or loses cell phone, he has to cancel the valid ID in the home information center to prevent illegal users from operating appliances, aiming at improving the system security. Because the user name and password are simple data types, user information can be stored by the Shared Preferences class, which stores private primitive data in key-value pairs.

\subsection{Hardware design}

This section mainly discuss about the hardware construction of main control board. Fig 2.2 demonstrates the hardware block diagram in the main control board. PIC Microcontroller, PIC18F2550 is chosen due to its capability to perform the both serial and USB features to establish the Bluetooth and USB connection to the GUIs. For the sensor, HSM-20G Sensor Module is chosen because it is the low cost 2-in-1 combination of humidity and temperature modules. For the Bluetooth module, low cost Cytron Bluebee Bluetooth module is chosen to establish the Bluetooth connection between main control board and the GUIs. 


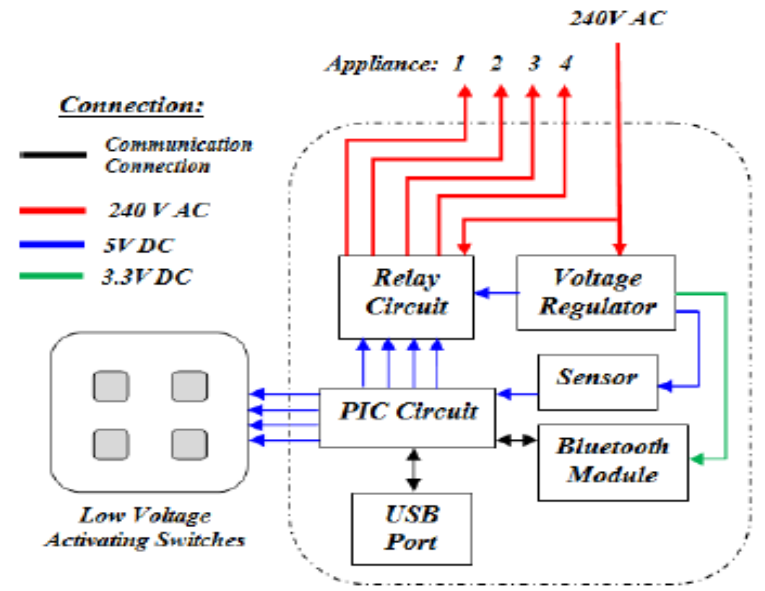

Fig 2.2 Main Control Board Hardware Block Diagram

The electrical current is directly connect to the main control board whereby it separates the regulator and relay circuit. The voltage regulator is constructed by common reliable regulator circuit which consists of transformer, rectifier and regulator. $5 \mathrm{~V}$ and $3.3 \mathrm{~V} \mathrm{DC}$ output is regulated in order to fulfill the voltage needs of the specific components in the main control board. Moreover, the low voltage activating switches will replace the existing switches

\subsection{Software design}

Figure 2.3 A show that the Bluetooth module and Android Smartphone connection flowchart, which show that transfer of data from Android application to Bluetooth Module after which is compare to programming code of AVR Microcontroller.

First check Bluetooth position of Android SmartPhone (Bluetooth is ON or OFF). If Bluetooth of android SmartPhone is ON then it check the surrounding Bluetooth Module and if Bluetooth Connection is off then user have to start the Bluetooth condition from OFF to ON of Android Smartphone. Then Smartphone search for the surrounding Bluetooth Module. After searching the Bluetooth Module it start pairing between Bluetooth Module and Android Smartphone. And if paring is occurs then hardware initialize the serial port. And Then PCB Hardware gets ready to receive the data (Character) which is send by the Android Application software.

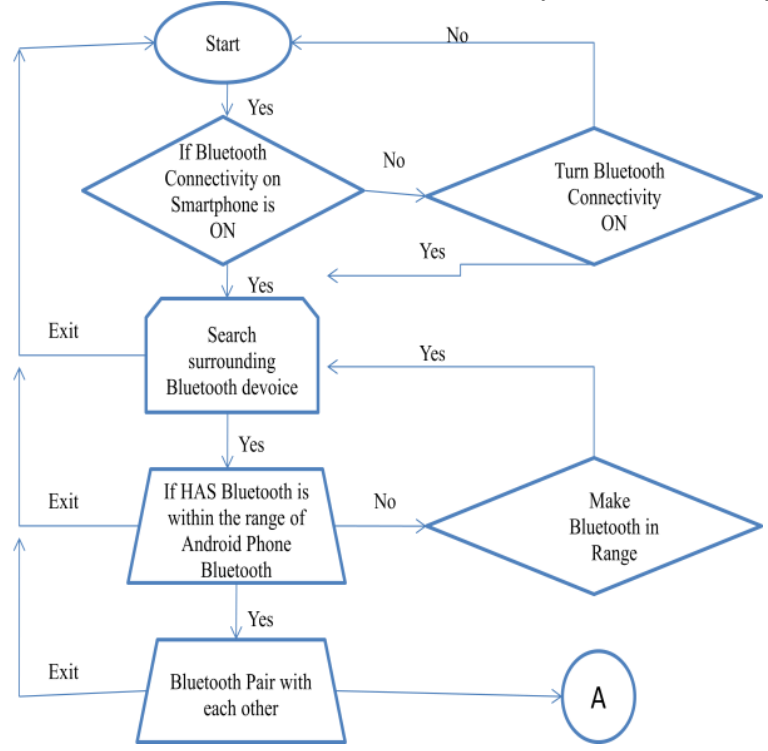

Fig.2.3.A Flowchart for Bluetooth Module and Android Smartphone connection flowchart 


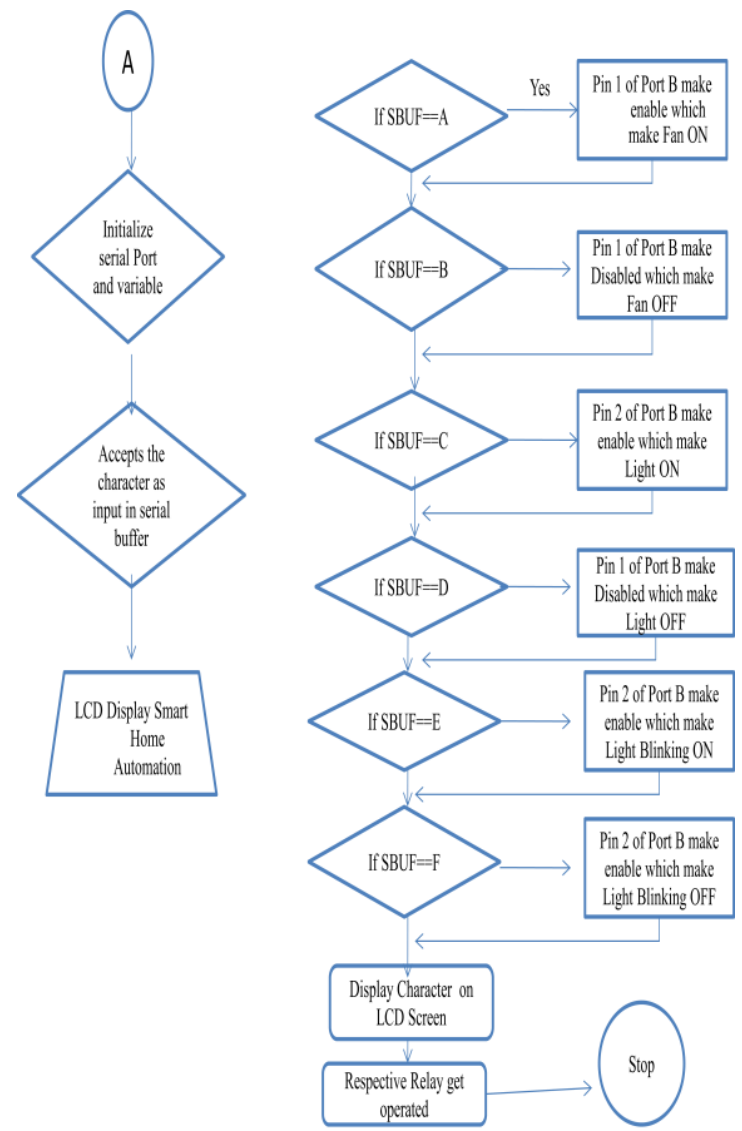

Fig.2.3.B Flowchart for relay control using Bluetooth Module

When the application software send the Text character to the microcontroller then this character get compare with the internal bourn code of the ATmega controller and accordingly the respective relay get ON and OFF. the passion of relay is depending on receiving character. if A or B is received by Bluetooth Receiver then Light is ON for turning On the UI button and Light OFF for Turning OFF the UI button. This is applicable for the entire button on the UI. Depending on the Button press respective character get send by Android Smartphone.

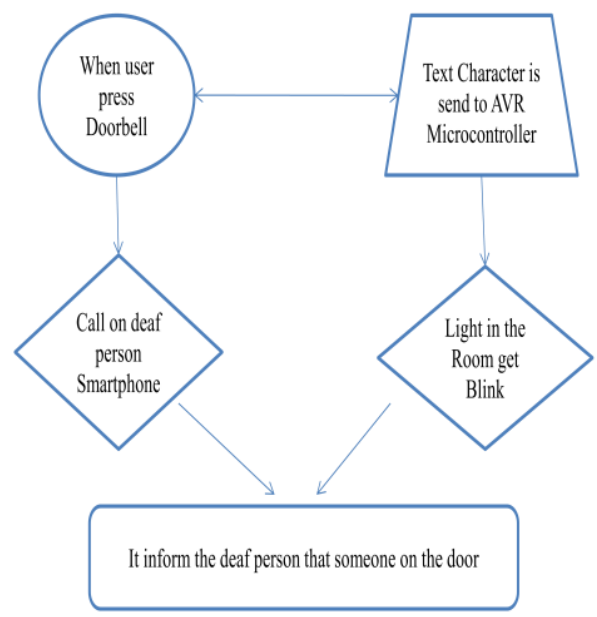

Fig.2.3.C GSM System Flowchart

After pressing the button of the doorbell (push Button) GSM system call on SmartPhone of deaf person as well as send character to AVR Microcontroller and depending of send character respective relay get on and off (Blinking)this two things inform that deaf person that is someone on the door or someone knock the door 


\subsection{Android Application}

To control the relays with the help of microcontroller relay is connected to the $4^{\text {th }}$ port of the PIC Microcontroller. By pressing the button on the GUI of the Android Smartphone we can control the relay.

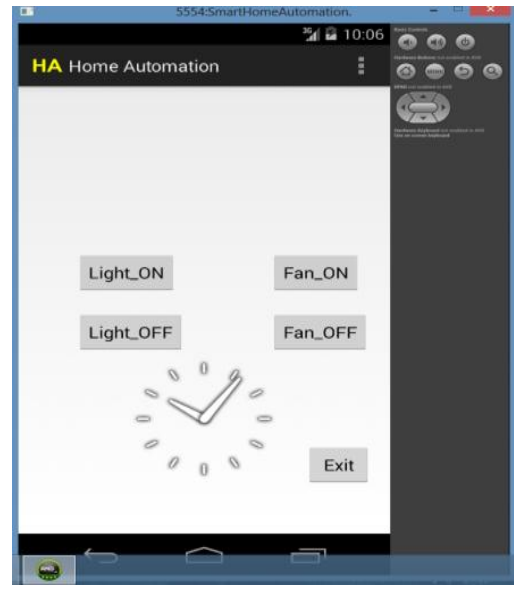

Fig.2.4. User Interface

Android's releases prior to $2.0(1.0,1.5,16)$ were used exclusively on Android mobile phones. Most Android SmartPhone and some Android tablets now use a 2.x release and Android 3.0 was a tablet-oriented release but does not officially run on mobile phones. The current Android version is 4.3. Android's releases are nicknamed after sweets or dessert items like Frozen Yogurt ("Froyo") (2.2), Ginger Bread (2.3), Honeycomb (3.0), Ice Cream Sandwich (4.0), Jelly Bean (4.1) and KitKat (4.4) being the recent one.

Android applications are written in the Java language. The Android Software Development Kit (SDK) provides all necessary tools to develop Android applications (API). This includes a compiler, debugger and a device emulator, as well as its own virtual machine (emulator) to run Android programs. The Android SDK provides tools for code compilation and packaging data and resource files into an archive file with '.apk' extension called as an Android package. Android devices used the '.apk' file to install the application. Android's application framework allows for the creation of extremely feature rich and novel applications by using a set of reusable components. The amalgamation of the Android development environment with the Bluetooth wireless technology is known by Android's support for the Bluetooth network stack, which permits a device to wirelessly exchange data with another Bluetooth device (SmartPhone Bluetooth with Bluetooth Module). The application framework enables access to the Bluetooth functionality using the Android Bluetooth APIs. These APIs allow wireless applications to connect to other Bluetooth devices for point-to-point and multipoint wireless features. Using the Bluetooth APIs, an Android application can carry out the following functions:

- Scrutinize for other Bluetooth devices

- Enquire about the local Bluetooth adapter for paired . Bluetooth devices

- Establish the RFCOMM channels

- Connect to other devices through service discovery.

- Exchange data to and from other devices

\subsection{Applications}

\section{Applications and Future Challenges}

Followings are the applications of home appliances control system based on the android SmartPhone.

\subsubsection{Lighting appliance control subsystem}

Household appliances also don't need to transmit large amounts of data, and it's real-time requirement is not high, so take a combination of wired and wireless ways to make wiring easy. Alarm subsystem with landline phone alarm and GSM alarm has multiple protections. It can still alarm normally when the line of the landline phone is cut off. The entire system takes modular design thinking which contributes to the design clear and facilitates the user to select a different combination of modules to meet the needs of individual users. Humanized operation interface allows users to use them more conveniently. Dimming control, a more comfortable environment and a certain scene was creating. Appliance control function is not only our commonly household appliances such as microwave ovens, water dispensers, rice cookers, television sets, but also includes garden automatic irrigation systems, fountains and other equipment. Lighting appliance control subsystem can 
be achieved not only a variety of romantic scenes, but also for the whole family safe. When you go out, just one lobby button, you can complete power outage, which significantly reduces the risk of fire and saves energy

\subsubsection{Curtain control subsystem}

Curtain control subsystem control all electric curtains including blinds, sunshades and skylights. It can automatically open and close in a particular time or at the intensity of light. You can control it by panel, remote control, and even the Internet and smart phones

\subsubsection{Future Challenges}

This project can be further developed by integrating it with the internet to monitor your home while sitting in a remote area. By doing this, one can keep an eye on his or her home through an internet connected to the user's mobile phone or PC or laptop. This will not only improve the security of your home in this modern day world but will also assist in conservation of energy like if you left any home appliance switched on by mistake, then you can check the status of the appliance on the graphical interface made on your mobile and can switch it off using the internet connectivity.

\section{Conclusions}

By designing the Android user interface and Home information centre, home appliance control system based on the Android phone can be designed. It has combined android client, network transmission, and wireless switch, home information center to form a complete system, and the whole system works normally. Identifying message commands and wireless encoding are the two major tasks for home information center. Android phone have advantages such as humane interface, customizable and extendible applications and android phone is easy to carry so on. By constantly improving the control function, android phone allows us anytime, anywhere to control any device, and finally realizes the highly intelligent home.

\section{References}

[1]. Jianjun Lv, Zhishu Li, Mingyi Mao. "A new USB home appliances based on PC and infrared remote control protocol".2010 International Conferences on Computer and Communication Technologies in Agriculture Engineering.2010, pp.572 -575

[2]. Shengwen Chen, Chunghuang Yang, Chung-Huang Yang."Design and Implementation of Live SD Acquisition Tool in Android Smart Phone". 2011 Fifth International Conference on Genetic and Evolutionary Computing. 2011, pp. 157-162.

[3]. Xiao Yuan, Yuliang Pan, Zaiying Ling. "The Application of Infrared Remote Controlled Code Lock in the Management of Industrial Machine Parameters” .Electrical and Control Engineering (ICECE), 2011 International Conference on.2011, pp. 418-421.

[4]. Feng Xun, Ye Zhi-xia."Sunplus SPCE061A MCU Simulation PT2262 Coding". Journal of Yunnan Normal University (Natural Sciences Edition), 2010. 30(4) . pp. 40-42.

[5]. Ki-Cheol Son, Jong-Yeol Lee. "The methods of android application speed up by using NDK". Awareness Science and Technology (iCAST), 2011 3rd International Conference on. Sept 2011, pp.382- 385. 